\title{
ĐỊNH VỊ TUYỆT ĐỐI KẾT HợP BA HỆ THỐNG GPS, GALILEO VÀ BEIDOU \\ NGUYẼ̃N NGỌC LÂUU( ${ }^{(1)}$ TRẦN VĂN NAM ${ }^{(2)}$ \\ (I) Truờng Đại học Bách khoa TP. Hồ Chí Minh \\ (2)Truờng Đại học Lâm nghiệp - Phân hiệu phía Nam
}

\section{Tóm tắt}

Nhằm muc đỉch nâng cao độ chính xác và khắc phuc nhũng khó khăn khi định vị GPS thông thuoòng không đạt kết quả tốt, chúng tôi đã nghiên cưu định vị tuyệt đối xử lý trị đo giả cư ly kết hợp ba hệ thống GPS, GALILEO và BEIDOU. Kết quả xủ lý tại 5 trạm đoIGS thuờng trực cho thấy độ chinh xác định vị huớng Đông, huớng Bắc và độ cao cải thiện so với định vị GPS thông thuờng lần luọt khoảng $63 \%, 65 \%$ và $60 \%$.

\section{Giới thiệu}

Định vị tuyệt đối GPS thông thường đang sử dụng phố biến nhất hiện nay. Nó có ưu điểm là xử lý đơn giản và áp dụng được cho đa số loại máy thu.Tuy nhiên, phương pháp có hạn chế là độ chính xác không cao, khoảng từ $5 \mathrm{~m}$ đến $15 \mathrm{~m}$. Hơn nữa, tại những khu vực có độ che phủ cao như gần nhà cao tầng, bìa rừng..., việc định vị nhiều khi không thực hiện được do số lượng vệ tinh cung cấp tín hiệu cho máy thu không đủ (< 4).

Để nâng cao độ chính xác định vị tuyệt đối, một trong số những biện pháp hiệu quả nhất là xử lý kết hợp nhiều hệ thống định vị vệ tinh khác nhau. Khi kết hợp, số lượng vệ tinh quan sát được tăng lên làm tăng số trị đo thừa, dẫn đến cải thiện độ chính xác. Mặt khác, định vị kết hợp có thể giải quyết vấn đề khó khăn tại những khu vực bị che khuất, mà định vị điểm sử dụng GPS thông thường không thực hiện được. Khó khăn của định vị kết hợp là phải có máy thu đa hệ thống vệ tinh, và cần giải quyết nhiều vấn đề liên quan sự khác nhau giữa các hệ thống định vị GNSS (Global Navigation Satellite Systems) như hệ toạ độ, thời gian, cấu trúc bản lịch vệ tinh, độ chính xác trị đo, các nguồn sai số hệ thống, .v.v...

Các hệ thống vệ tinh định vị đã và đang phát triển hiện nay bao gồm GPS, GALILEO, BEI-
DOU và GLONASS. Cả bốn hệ thống định vị có nhiều đặc điểm tương tự nhau, tuy nhiên trong đó ba hệ thống GPS, GALILEO và BEIDOU có nhiều điểm tương đồng với nhau nhiều nhất. Đây là những thuận lợi trong việc xử lý tích hợp. Hệ thống GPS, GALILEO và BEIDOU sử dụng kỹ thuật đa truy cập phân chia theo mã (Code Division Multiple Access - CDMA)có phép nhiều vệ tinh sử dụng chung các tần số giống nhau. Trong khi hệ thống GLONASS sử dụng kỹ thuật phân chia theo tần số (Frequency Division Multiple Access - FDMA) nên mỗi vệ tinh bắt buộc phải truyền tín hiệu theo những tần số khác nhau. Trong nghiên cứu này, chúng tôi đã thực hiện định vị tuyệt đối kết hợp trị đo giả cự ly của ba hệ thống GPS, GALILEO và BEIDOU.

Để giải quyết những vấn đề xử lý tích hợp, trong bài báo này, chúng tôi lần lượt trình bày việc khắc phục các nguồn sai sốdo ảnh hưởng của khí quyển, sai số đồng hồ máy thu, sai số giữa các hệ thống định vị (Inter-system Bias ISB)... Tiếp theo chúng tôi khảo sát bộ trọng số trị đo giả cự ly cho các hệ thống GALILEO và BEIDOU tương ứng với GPS. Cuối cùng, việc xử lý tích hợp ba hệ thống có áp dụng bộ trọng số khảo sát được tại 5 trạm đo khác nhau và chứng minh sự cải thiện độ chính xác kết hợp GPS, GALILEO và BEIDOU so với định vị GPS thông thường. 


\section{Những vấn đề cần giải quyết khi xử lý kết hợp}

2.1. Chuyển đổi về cùng hệ tọ̣ độ và hệ thống thời gian

Để thực hiện bài toán định vị kết hợp cần đưa các hệ thống về cùng một hệ toạ độ và hệ thời gian. Trong nghiên này cứu chúng tôi đưa các hệ thống về cùng hệ toạ độ WGS84 và hệ thống thời gian GPST của GPS.

Như đã biết,GPS sử dụng hệ toạ độ WGS84, đồng nhất với hệ ITRF2008 thời điểm 2000 [5]. Hệ thống GALILEO sử dụng hệ toạ độ GTRF. Phiên bản mới nhất của hệ thống toạ độ GALILEO là GTRF13v02 đồng nhất với ITRF2008 và sai lệch khoảng $3 \mathrm{~cm}$ [8]. Hệ thống BEIDOU sử dụng hệ toạ độ CGCS2000 đồng nhất với ITRF97 tại thời điểm 2000 [9].

Để chuyển đổi từ hệ toạ độ này về hệ toạ độ kia chúng ta sử dụng bài toán 7 tham số. Theo website của tổ chức ITRS giữa các phiên bản khác nhau của hệ toạ độ ITRF có sự sai lệch chỉ ở mức $\mathrm{cm}$. Trong nghiên cứu này, chúng tôi quan tâm đến độ chính xác định vị ở mức vài dm- mét nên việc ảnh hưởng nhỏ do khác biệt giữa các phiên bản ITRF có thể bỏ qua.

Đối với hệ thống thời gian, hệ thống GALILEO sử dụng hệ thống thời gian GST (GALILEO Time) trùng với hệ thống thời gian của GPS [5].Hệ thống BEIDOU sử dụng hệ thống thời gian BDT(BEIDOU Time) lệch so với GPST là 14 giây, BDT = GPST - 14 giây.

\subsection{Khắc phục các nguồn sai số trong trị đo giả cụ ly}

Phương trình trị đo giả cự ly GNSS có dạng tổng quát như sau [4]:

$$
P^{i}=p^{i}+c .\left(d t-d T^{i}\right)-I^{i}+T^{i}+c . I S B
$$

Trong đó:

$P^{i}$ : Là trị đo giả cự ly ứng với vệ tinh thứ $i$

$p^{i}$ : Khoảng cách hình học từ vệ tinh $i$ đến máy thu

$$
\rho^{i}=\sqrt{\left(X_{i}-X_{r}\right)^{2}+\left(Y_{i}-Y_{r}\right)^{2}+\left(Z_{i}-Z_{r}\right)^{2}}
$$

Với : $X_{i}, Y_{i}, Z_{i}$ là toạ độ vệ tinh thứ $\mathrm{i}$

$X_{r}, Y_{r}, X_{r}$ là toạ độ gần đúng của máy thu

c vận tốc ánh sáng trong môi trường chân không

dt là số hiệu chỉnh đồng hồ máy thu

$d T^{i}$ số hiệu chỉnh đồng hồ vệ tinh

$I^{i}$ độ trễ điện ly

$T$ độ trễ đối lưu

ISB độ trễ hệ thống do quá trình giải mã tín hiệu của máy thu GNSS đến từ các vệ tinh không cùng hệ thống. Số hạng này chỉ tồn tại trong các trị đo GALILEO và BEIDOU, còn đối với GPS thường giả sử bằng 0 .

Phương trình (1) cho thấy trị đo GNSS có chứa 5 nguồn sai số chính.Phương pháp để khử hoặc giảm các nguồn sai số như sau:

- Khắc phục ảnh hưởng của tầng điện ly

Đối với máy thu một tần số nguồn sai số này được hiệu chỉnh theo các mô hình kinh nghiệm. Ví dụ mô hình Klobuchar có thể loại bỏ khoảng $60 \%$ ảnh hưởng điện ly. Đối với máy thu 2 tần số, người ta sử dụng phương pháp kết hợp trị đo tạo ra trị đo $\mathrm{P}_{3}$.Trị đo này loại bỏ hơn $90 \%$ ảnh hưởng của điện ly [4]. Trị đo $\mathrm{P}_{3}$ được tính theo công thức sau:

$$
P_{3}^{i}=\alpha_{1} P_{1}^{i}+\alpha_{2} P_{2}^{i}=\rho^{i}+c .\left(d t-d T^{i}\right)+T^{i}+c . I S B
$$

Trong đó: $P_{1}^{i}$ và $P_{2}^{i}$ là các trị đo giả cự ly ở hai tần số $\mathrm{f}_{\mathrm{L} 1}$ và $\mathrm{f}_{\mathrm{L} 2}$

$$
\alpha_{1}=\frac{\left(f L_{2}\right)^{2}}{\left(f L_{1}\right)^{2}-\left(f L_{2}\right)^{2}} \text { và } \alpha_{2}=\frac{\left(f L_{2}\right)^{2}}{\left(f L_{L_{1}}\right)^{2}-\left(f L_{L_{2}}\right)^{2}}
$$

- Khắc phục ảnh hưởng của tầng đối lưu

Tầng đối lưu là tầng khí quyển tính từ mặt đất đến độ cao khoảng $50 \mathrm{~km}$. Trong đó tầng đối lưu chứa nhiều hơi nước và bụi khí quyển. Ảnh hưởng của tầng đối lưu đến tín hiệu điện từ không phụ thuộc vào tần số sóng tải, được chia 
thành ảnh hưởng của phần khô (trên cao) và ảnh hưởng của phần ướt (dưới thấp). Ảnh hưởng của phần khô chiếm khoảng $90 \%$ và ảnh hưởng của phần ướt là $10 \%$ [2].

Chúng tôi tính độ trễ đối lưu hướng thiên đỉnh theo công thức thường dùng nhất hiện nay là Saastamoinen, công bố vào năm 1973 [4].Để chuyển từ hướng thiên đỉnh sang hướng bất kỳ phải chọn hàm ánh xạ (mapping function). Hàm ánh xạ được sử dụng phổ biến hiện nay của Niell công bố năm 1996 [4]. $I S B$

- Khắc phục sai số đồng hồ máy thu và sai số

Sai số ISB được hình thành do việc xử lý tín hiệu không đồng bộ của máy thu khi thu nhận tín hiệu từ các hệ thống định vị vệ tinh khác nhau.Sai số này chủ yếu xuất hiện trọng hệ thống GALILEO và $B E I D O U$ với độ lớn khác nhau và phụ thuộc vào kiểu máy thu. ISB sẽ ảnh hưởng đáng kể đến kết quả bài toán định vị kết hợp trị đo các hệ thống khác nhau.Vì vậy nó cần được loại bỏ khỏi trị đo trước khi đưa vào bài toán định vị.

Để khử thành phần sai số máy thu và sai số ISB, ta cần lấy hiệu nộ $i$ bộ các trị đo giả cự ly của từng hệ thống [4]. Nghĩa là mỗi hệ thống định vị chọn một vệ tinh làm chuẩn và lấy hiệu với các vệ tinh khác trong cùng hệ thống. Phương trình hiệu trị đo giả cự ly giữa vệ tinh $k$ và vệ tinh $l$ của cùng một hệ thống tại máy thu là:

$$
\Delta P_{3}^{k l}=\Delta \rho^{k l}-c \cdot \Delta d T^{k l}+\Delta T^{k l}
$$

Như vậy, phương trình (5) đã giảm hoặc loại bỏ các nguồn sai số do ảnh hưởng tầng điện ly, sai số đồng hồ máy thu và sai số ISB. Trong phương trình giả cự ly chỉ còn lại hiệu sai số đồng hồ vệ tinh và hiệu độ trễ đối lưu.Sai số đồng hồ vệ tinh được tính từ thông báo hàng hải của từng hệ thống định vị, còn độ trễ đối lưu được tính theo mô hình Saastamoinen và hàm ánh xạ Niell.

\section{thống}

Các hệ thống định vị khác nhau sẽ cung cấp trị đo giả cự ly có độ chính xác không giống nhau. Nếu xem chúng có độ chính xác như nhau, tức là có trọng số trị đo bằng nhau, kết quả xử lý tích hợp có khi sẽ kém chính xác hơn định vị GPS thông thường. Vì vậy vấn đề xác trọng số trị đo thích hợp cho từng hệ thống rất quan trọng. Để xác định trọng số cho từng hệ thống, chúng tôi dựa vào công thức [4]:

$$
p_{i}=p_{0} \sin ^{2} \varepsilon_{i}
$$

Trong đó: $\varepsilon_{\mathrm{i}}$ là góc cao của vệ tinh $i, p_{i}$ là trọng số trị đo vệ tinh thứ i, và $p_{0}$ là giá trị trọng số đơn vị.

Dùng (6) ta lập công thức tính trọng số của hệ thống GPS và GALILEO/BEIDOU như sau:

$$
\begin{aligned}
& p_{G P S}=p_{0}^{G P S} \sin ^{2} \varepsilon \\
& p_{G L L / B D S}=p_{0}^{G L L / B D S} \sin ^{2} \varepsilon
\end{aligned}
$$

Để khảo sát tìm trọng số đơn vị $\mathrm{p}_{0}$ của từng hệ thống định vị, chúng ta cần có toạ độ chính xác máy thu, trị đo các hệ thống, toạ độ vệ tinh và sai số đồng hồ vệ tinh. Việc khảo sát trọng số được thực hiện theo cácbước sau:

Buớc 1: Cố định tọa độ máy thu vào giá trị chính xác

$$
\text { Bước 2: } \quad \text { Chop } p_{0}^{G P S}=p_{0}^{G L L / B D S}=1
$$

Bước 3: Tính phần dư trị đo là hiệu giữa giá trị của trị đo với giá trị mô hình

$$
\begin{aligned}
& v_{G P S}=p_{\text {trido }}^{\text {GPS }}-p_{M H}^{G P S} \\
& v_{G L L / B D S}=p_{\text {trido }}^{\text {GLL/BDS }}-p_{M H}^{G L L / B D S}
\end{aligned}
$$

Trong đó:

$v_{G P S}, v_{G L L / B D S}$ là phần dư trị đo của hệ thống GPS và GALILEO/BEIDOU

$P_{\text {trido }}^{G P S}, P_{\text {trido }}^{G L L / B D S}$ là trị đo giả cự ly kết hợp $\mathrm{P}_{3}$ của GPS và GALILEO/BEIDOU

$P_{M H}^{G P S}, P_{M H}^{G L L / B D S}$ là các giá trị mô hình được tính từ công thức $\mathrm{P}_{\mathrm{MH}}=\rho_{0}{ }^{\mathrm{i}}+\mathrm{T}^{\mathrm{i}}+\mathrm{c} \cdot\left(\mathrm{dt}-\mathrm{dT} \mathrm{T}^{\mathrm{i}}\right)(10)$ 
$\rho_{0}{ }^{\mathrm{i}}$ là khoảng cách hình học từ vệ tinh $\mathrm{i}$ đến máy thu, được tính theo toạ độ chính xác máy thu

$T^{\mathrm{T}}$ là độ trễ đối lưu của vệ tinh i tính theo mô hình Sasstamoinen và hàm ánh xạ Neill

$d T$ sai số đồng hồ vệ tinh i tính từ bản lịch phát tín

Bước 4: Tính sai số trung phương trọng số đơn vị

$$
\text { a. Hệ thống GPS: } \mu_{G P S}=\sqrt{\frac{V_{G P S}^{T} S^{P} G P V_{G P S}}{n_{1}-k}}
$$

Trong đó: $n_{1}$ là số lượng trị đo GPS, và $k$ là số lượng ẩn số

b. Hệ thống GLL/BDS:

$$
\mu_{G L L / B D S}=\sqrt{\frac{V_{G L L / B D S^{P} G L L / B D S V_{G L L / B D S}^{T}}^{n_{2}-k}}{n_{2}}}
$$

Trong đó: $n_{2}$ là số lượng trị đo GALILEO/BEIDOU

Bước 5: Tính hệ số $K=\frac{\mu G P S}{\mu_{G L L / B D S}}$

Ta có trọng số của GALILEO:

$$
p_{0}^{G L L / B D S}=p_{0}^{G P S} \cdot K^{2}
$$

Bước 6: Lặp lại từ bước 3 cho đến khi $K=1$ thì dừng lại.

Kết quả khảo sát trọng số thực hiện tại 5 trạm đo gồm HCMC, ARUC, HKWS, IISC và JFNG. Thông tin về các trạm này sẽ giới thiệu chi tiết ở muc 3. Kết quả tính trọng số GALILEO và BEIDOU trình bày ở bảng 1 .

Nhằm tăng "tính khách quan" cho trọng số chúng tôi đã lấy trung bình các giá trị trọng số của 5 trạm khảo sát được

$$
p_{0}^{\text {GPS }}=1, p_{0}^{\text {GALILEO }}=13.13, p_{0}^{\text {BEIDOU }}=0.19 .
$$

Các giá trị trọng số này là trọng số đại diện cho các trị đo của GPS, GALILEO và BEIDOU. Qua kết quả khảo sát trọng số chỉ ra rằng độ chính xác của hệ thống GPS kém hơn hệ thống GALILEO nhưng cao hơn hệ thống BEIDOU.

\section{Dữ liệu thực nghiệm}

Dữ liệu GNSS dùng cho nghiên cứu bao gồm toạ độ chính xác của trạm đo, file bản lịch phát tín, file trị đo của các hệ thống ở định dạng RINEX. Hiện nay có trên 200 trạm GNSS thường trực phân bố khắp thế giới. Tuy nhiên, phần lớn các trạm không có số liệu của hệ thống BEIDOU, hoặc số lượng vệ tinh GALILEO/BEIDOU thu được quá ít $(<4)$. Do đó, chúng tôi đã chọn lọc được 5 trạm phân bố chủ yếu ở khu vực Châu Á vào ngày 11 tháng 8 năm 2018. Các trạm bao gồm HCMC ở thành phố Hồ Chí Minh - Việt Nam, trạm ARUC thuộc nước Armenia, trạm HKWS đặt tại Wong Shek thuộc Hồng Kông, trạm IISC ở thành phố Bangalore thuộc Ấn Độ, trạm JFNG ở thành phố Vũ Hán thuộc Trung Quốc. Vị trí các trạm khảo sát được mô tả ở hình 1 .

Giá trị toạ độ chính xác của các trạm IGS dựa trên kết quả xử lý hàng tuần. Các toạ độ này được cho trong ITRF14 và có độ chính xác vài milimet.

\section{Kết quả và phân tích}

Chúng tôi sử dụng thuật toán, dữ liệu thu thập được và trọng số khảo sát được trình bày ở muc 2 và 3. Dùng ngôn ngữ Matlab, chúng tôi viết

Bảng 1: Kết quả trọng số trị đo giả cự ly của hệ thống GALILEO và BEIDOU

\begin{tabular}{|c|c|c|c|c|c|c|}
\hline Trậ thống đo & HCMC & ARUC & HKWS & IISC & JFNG & Trung bình \\
\hline GALILEO & 9.72 & 11.21 & 16.00 & 9.11 & 19.57 & $\mathbf{1 3 . 1 3}$ \\
\hline BEIDOU & 0.25 & 0.18 & 0.20 & 0.12 & 0.22 & $\mathbf{0 . 1 9}$ \\
\hline
\end{tabular}


chương trình định vị tuyệt đối có khả năng xử lý riêng cho từng phương án sau đây:

- Chỉ dùng duy nhất trị đo GPS

- Kết hợp trị đo GPS và BEIDOU

- Kết hợp trị đo GPS và GALILEO

- Kết hợp trị đo GPS, GALILEO và BEIDOU

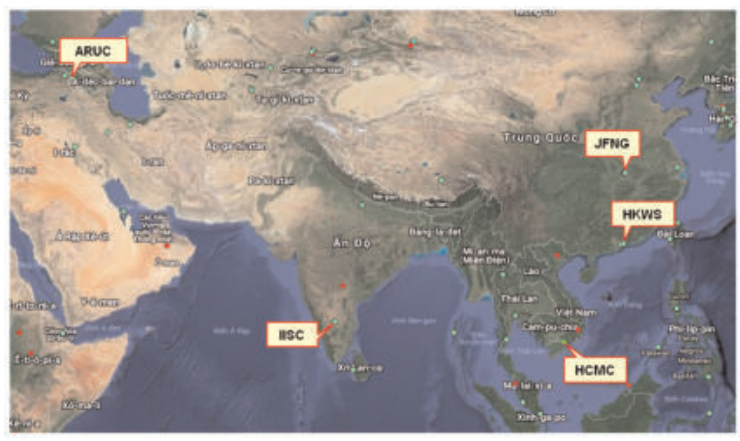

Hình 1: So đồ vị trí các trạm khảo sát

Kết quả từng trạm và trung bình của 5 trạm khảo sát được trình bày tại bảng 2 . Trong đó thể hiện sai số trung phương theo hướng Bắc, hướng Đông, độ cao, sai số mặt bằng và sai số không gian ( 3 chiều) lần lượt là $\mathrm{m}_{\mathrm{N}}, \mathrm{m}_{\mathrm{E}}, \mathrm{m}_{\mathrm{U}}, \mathrm{m}_{2 \mathrm{D}}, \mathrm{m}_{3 \mathrm{D}}$. Kết quả này cho thấy độ chính xác định vị tăng theo số lượng hệ thống vệ tinh định vị. Sự cải thiện rõ rệt nhất là ở thành phần hướng Bắc. Biểu đồ hình 2 so sánh kết quả sai số tổng hợp ở các phương án định vị. Đều là phương án kết hợp hai hệ thống, nhưng khi kết hợp GPS+GALILEO cho độ chính xác cao hơn kết hợp GPS+BEIDOU. Nguyên nhân chính là do trị đo GALILEO có độ chính xác cao hơn BEIDOU.

Khi so sánh với định vị chỉ sử dụng trị đo GPS, độ chính xác định vị 3 chiều của phương án kết hợp GPS+BEIDOU được cải thiện 15\%, phương án kết hợp GPS+GALILEO cải thiện 59\%, phương án kết hợp ba hệ thống GPS+GALILEO+BEIDOU là $61 \%$.

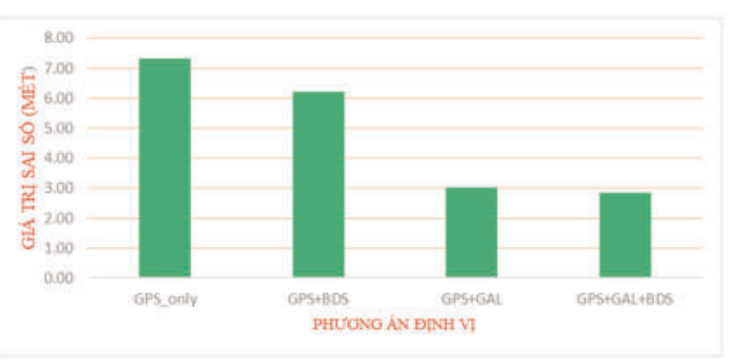

Hình 2: So sánh độ chính xác giũ̃a các phuoong án định $v i$

\section{Kết luận}

Chúng tôi đã trình bày phương pháp định vị tuyệt đối kết hợp trị đo của ba hệ thống GPS, GALILEO và BEIDOU để nâng cao độ chính xác so với định vị sử dụng GPS. Sử dụng ngôn ngữ Matlab, chúng tôi viết chương trình xử lý định vị tuyệt đối kết hợp ba hệ thống định vị. Kết quả xử lý tại 5 trạm IGS cho thấy:

- Độ chính xác định vị được cải thiện theo số lượng hệ thống vệ tinh tham gia trong bài toán định vị kết hợp. Độ chính xác định vị không gian khi kết hợp GPS+GALILEO+BEIDOU là

Bảng 2: Kết quả định vị tuyệt đối ở các phưong án khác nhau

\begin{tabular}{|c|c|c|c|c|c|c|}
\hline \multirow{2}{*}{$\begin{array}{c}\text { Phương án } \\
\text { định vị }\end{array}$} & \multicolumn{5}{|c|}{ Sai số trung phương (m) } & \multirow{2}{*}{$\begin{array}{c}\text { Cải } \\
\text { thiện } \\
(\%)\end{array}$} \\
\hline & $\mathbf{m}_{N}$ & $\mathbf{m}_{\mathbf{E}}$ & $\mathbf{m}_{\mathbf{U}}$ & $\mathbf{m}_{2 \mathrm{D}}$ & $\mathbf{m}_{3 \mathrm{D}}$ & \\
\hline GPS only & 2.75 & 2.68 & 6.20 & 3.83 & 7.30 & - \\
\hline GPS+BEIDOU & 2.47 & 2.46 & 5.12 & 3.49 & 6.19 & 15 \\
\hline GPS+GALILEO & 1.06 & 1.07 & 2.60 & 1.51 & 3.00 & 59 \\
\hline GPS+GALILEO+BEIDOU & 0.95 & 1.00 & 2.49 & 1.38 & 2.85 & 61 \\
\hline
\end{tabular}


$2.85 \mathrm{~m}$, cải thiện $61 \%$ so với định vị GPS thông thường. Trong đó, độ chính xác về mặt bằng hướng Đông đạt $1.00 \mathrm{~m}(63 \%)$, hướng Bắc $0.95 \mathrm{~m}(65 \%)$ và độ cao $2.49 \mathrm{~m}(60 \%)$.

- Do độ chính xác trị đo của GALILEO tốt hơn BEIDOU, kết hợp GPS+GALILEO vì vậy cũng cho độ chính xác định vị tốt hơn GPS+BEIDOU.

Các kết quả trên chỉ sử dụng 5 trạm đo cho một ngày dữ liệu 24h. Để nhận được kết quả có tính chất thống kê hơn nữa, trong tương lai chúng tôi sẽ tiếp tục xử lý tập dữ liệu lớn hơn về số lượng và phân bố không gian rộng hơn khi hệ thống BEIDOU tiếp tục mở rộng ảnh hưởng của mình trên phạm vi toàn cầu. $\bigcirc$

\section{Tài liệu tham khảo}

[1]. Đỗ Công Hữu, (2015), Nghiên cưu định vị điểm kết hơp vệ tinh GPS và SBAS, Luận văn thạc sĩ, Trường Đại học Bách Khoa TP. Hồ Chí Minh.

[2]. Đặng Nam Chinh, (2012), Định vị vệ tinh, Nhà xuất bản Khoa học và Kỹ thuật.

[3]. Jari Nurmi, Elena Simona Lohan, Stephan Sand, Heikki Hurskainen,
(2015),GALILEO Positioning Technology, Springer Netherlands.

[4]. Nguyễn Ngọc Lâu, Bài giảng GPS nâng cao, Đại học Bách Khoa TP Hồ Chí Minh.

[5]. Nguyễn Ngọc Lâu, (2013), Định vị điểm chính xác tích hợp trị đo GPS và GLONASS, Tạp chi khoa hoc Đo đạc và Bản đồ, số 15, pp. 9-7.

[6]. Nguyễn Ngọc Lâu, (2009), Định vị điểm đơn bằng GPS hiện nay có thể đạt độ chính xác bao nhiêu?, Tạp chí phát triển KH\&CN, tập 12, số 08, pp. 39-44.

[7]. Nguyễn Ngọc Lâu, Nguyễn Đăng Thiện, (2001), Đánh giá độ chính xác các thông tin trong bản lịch phát tín GPS sau khi SA tắt, Tạp chí Trắc địa-Bản đồ, số 1, pp. 38-44.

[8]. ESA Galileo Terrestrial Reference Frame 11/2013.

[9]. Nguyễn Ngọc Lâu, (2016), Thử nghiệm định vị tuyệt đối dùng hệ thống vệ tinh BEIDOU tại Việt Nam, Tạp chí Phát triển KH\&CN, Tập 19, số K4-2016, pp. 19-26. O

\section{Summary}

\section{Absolute positioning using combined GPS, GALILEO and BEIDOU observations}

Nguyen Ngoc Lau, Ho Chi Minh City University of Technology

Tran Van Nam, Vietnam National University of Forestry (southern campus)

In order to improve accuracy and overcome difficulties when GPS positioning does not provide good result, we have researched absolute positioning processing mixed pseudo-ranges of three GPS, GALILEO and BEIDOU systems. The processing results at 5 IGS permanent stations show that the positional accuracies of the East, North and Up components are improved compared to GPS only case of about $63 \%, 65 \%$ and $60 \%$ respectively. $\bigcirc$ 